\title{
PROGRAMA EDUCACIONAL PARA UNIDADES DE TERAPIA INTENSIVA NEONATAIS E PEDIÁTRICAS BRASILEIRAS
}

\author{
EDUCATIONAL PROGRAM FOR BRAZILIAN NEONATAL AND PEDIATRIC INTENSIVE CARE UNITS
}

\author{
Edna Aparecida Bussotti ${ }^{1}$ \\ Eliseth Ribeiro Leão ${ }^{2}$ \\ Kellen Cristensen ${ }^{3}$ \\ Luiz Maria Ramos Filho ${ }^{4}$ \\ Teresa Maria Uras Belem ${ }^{5}$ \\ Katia Jarandilha dos Santos ${ }^{6}$
}

Resumo Trata-se de um estudo descritivo e exploratório que objetivou avaliar os padrões de qualidade em saúde em 13 unidades de terapia intensiva neonatal e pediátrica da rede pública brasileira nos períodos anteriores (diagnóstico situacional) e posteriores (reavaliação) ao desenvolvimento do Programa de Apoio ao Desenvolvimento Institucional do Sistema Único de Saúde. Foi elaborado um instrumento para avaliação diagnóstica com base na portaria n. 3.432, de 12 de agosto de 1998. A capacitação foi planejada mediante o diagnóstico situacional. Foram mensurados dez critérios de avaliação e, na fase diagnóstica, cinco apresentavam $40 \%$ de índice de conformidade. O programa de capacitação foi estruturado em treinamento on the job (12 horas), totalizando 290 horas, e curso com visitas monitoradas (30 horas), totalizando 810 horas/homem e média de 180 horas/homem. Na reavaliação houve elevação dos índices em nove dos dez critérios avaliados. A metodologia adotada possibilitou o desenvolvimento de um programa educacional com base no diagnóstico situacional, com indicadores de treinamento satisfatórios, considerando a peculiaridade do setor público de saúde. A participação dos profissionais do hospital de excelência foi relevante para o alcance dos resultados, proporcionando espaços para discussão e troca de experiências.

Palavras-chave responsabilidade social; Sistema Único de Saúde; educação continuada; indicadores de gestão; qualidade da assistência à saúde.
Abstract This is an exploratory and descriptive study that aimed to evaluate the quality standards in health in 13 Brazilian public neonatal and pediatric intensive care units in periods prior (situational diagnosis) and subsequent (reassessment) to the development of the Program for Support for the Institutional Development of the Unified Health System. A diagnostic assessment tool was prepared based on decree n. 3432, of August 12, 1998. Training was planned based on the situational diagnosis. Ten assessment criteria were measured and, in the diagnostic phase, five had a 40 percent compliance rate. The training program was structured with on-the-job training (12 hours), which added up to 290 hours, and on a course with guided visits (30 hours), totaling 810 man hours and with an average of 180 man hours. During the reassessment, it was noted that there was an increase in nine of the ten criteria evaluated. The methodology that was adopted enabled the development of an educational program based on situational analysis, with satisfactory training indicators, considering the peculiarity of the public health sector. The participation of professionals from the hospital of excellence was relevant to the achievement of the results, as this provided space for discussion and for the exchange of experiences.

Keywords social responsibility; Unified Health System; continuing education; management indicators; health care quality. 


\section{Introdução}

No Brasil, os índices de mortalidade infantil estão em declínio desde 1998, mas os dados de mortalidade nas regiões Norte e Nordeste ainda continuam altos quando comparados às demais regiões e ao índice geral do país. A mortalidade infantil decorre de diversos fatores: biológicos, sociais, culturais, educacionais e de falhas do sistema de saúde. Sendo assim, as intervenções dirigidas à sua redução dependem de mudanças estruturais relacionadas às condições de vida da população, bem como de ações diretas definidas por políticas públicas de saúde. Dentre as maiores causas da mortalidade neonatal, destacam-se: prematuridade, infecções, asfixia/hipóxia, malformações congênitas, fatores maternos e relacionados à gravidez, e afecções respiratórias do recém-nascido (França e Lansky, 2009).

Em relação às causas de morte mais observadas no período pós-neonatal, destacam-se as doenças respiratórias e as doenças do trato gastrointestinal (doenças diarreicas), cujo fator de risco identificado foi referente às diferenças socioeconômicas regionais (Santos et al., 2010).

Ao se analisarem os índices e as causas relacionadas à mortalidade infantil, fica evidenciado que o subgrupo classificado como 'mortes evitáveis' apresenta números alarmantes. No ano de 2010, a taxa de mortes evitáveis do total de óbitos entre neonatos (desde a primeira hora de vida) e crianças com até 1 ano de idade foi de $61 \%$. Tal evidência alerta para a necessidade de se verificar a qualidade e a segurança da assistência prestada à população (Brasil, 2002; Abreu, César e França, 2007)

Dentre as causas de mortes evitáveis na população infantil, destacam-se a falta de atenção adequada à mulher durante a gestação e no intra e no pós-parto, de diagnóstico e tratamento adequados aos neonatos e crianças e de ações de promoção à saúde para esses segmentos populacionais (Abreu, César e França, 2007; Malta et al., 2007).

Para o adequado atendimento dos agravos à saúde das populações neonatal e pediátrica são necessárias unidades de cuidados intensivos com profissionais capacitados, estrutura adequada e processos bem estabelecidos.

No entanto, com o avanço técnico, tecnológico e de recursos humanos dos últimos vinte anos, as unidades de terapia intensiva neonatal (Utins) e as unidades de terapia intensiva pediátrica (Utips) se depararam com um aumento de internações de crianças em condições cada vez mais graves e, no caso das Utins, de crianças mais prematuras, que dependem de tecnologias e recursos humanos especializados (Barbosa, 2004; Souza et al., 2004). O número crescente de crianças que necessitam de unidades de terapia intensiva (UTIs) levou ao crescimento de muitos serviços na tentativa de atender tal demanda, e esses serviços continuam crescendo, de forma desordenada e sem um planejamento estratégico adequado (Barbosa, 2004). E isso 
mesmo que tenham sido publicadas portarias e legislações (por exemplo, a portaria n. 3.432, do Ministério da Saúde, de agosto de 1998, e a resolução da Agência de Vigilância Sanitária RDC n. 50, de 21 de fevereiro de 2002) determinando as boas práticas de funcionamento das Utins e Utips com os requisitos de segurança e qualidade no atendimento às crianças.

A vulnerabilidade desse segmento populacional é um indicativo da necessidade de unidades especializadas e de profissionais capacitados para que se alcance a melhor performance terapêutica possível. Segundo a Sociedade Brasileira de Pediatria (SBP), a necessidade de procedimentos de reanimação do neonato é maior quanto menor a idade gestacional ou o peso ao nascer. No Brasil, a cada ano, 300 mil crianças necessitam de auxílio para manter a respiração ao nascer, e cerca de 25 mil prematuros necessitam de ventilação mecânica na sala de parto, o que repercute diretamente no seguimento da assistência oferecida na Utin. Com o avanço tecnológico, os diagnósticos, que são feitos mais precocemente, passaram a implicar novas necessidades terapêuticas que atendam essas demandas. Todavia, ainda evidenciamos no Brasil, profissionais pouco qualificados para o atendimento específico de populações tão vulneráveis (Almeida e Guinsburg, 2011).

Pesquisas apontam para a importância da capacitação de recursos humanos a fim de se atingirem melhorias na prática assistencial, diminuindo os riscos de eventos adversos e, consequentemente, aumentando de forma positiva os resultados assistenciais e financeiros. Assim, nos serviços de saúde, a gestão de pessoas deve prever o desenvolvimento de competências técnicas para assegurar a melhoria contínua. A capacitação de pessoas é um dos recursos necessários para atingir metas e promover mudanças de resultados, bem como a avaliação dos programas educacionais e o desenvolvimento de pesquisas relacionadas às tecnologias mediadas por informação (Nepomuceno e Kurcgant, 2008; Ceccim e Feuerwerker, 2004), cada vez mais úteis em um país de dimensões continentais como o Brasil. O Ministério da Saúde, por meio da portaria n. 1.996, de 20 de agosto de 2007, dispõe sobre as diretrizes para a implantação da Política Nacional de Educação Permanente em Saúde e dá outras providências. Seu objetivo é fornecer a base normativa adequada para a organização dos processos de gestão da educação na saúde, nas diferentes esferas de gestão.

O documento enfatiza a educação permanente como aprendizagem no trabalho, pela qual o aprender e o ensinar se incorporam ao cotidiano das organizações e ao trabalho. A educação permanente se baseia na aprendizagem significativa e na possibilidade de transformar as práticas profissionais. Devem-se considerar os problemas enfrentados na realidade, os conhecimentos e as experiências previamente adquiridos. Os processos de educação permanente em saúde (EPS) têm como objetivos a transformação das práticas profissionais e da própria organização do trabalho. Profissionais 
qualificados certamente contribuem com resultados positivos para o paciente, a família e, consequentemente, para a própria instituição.

A qualidade assistencial e a segurança do paciente são temas que não apenas despertam a atenção do cidadão em geral, mas também constituem foco de árduo trabalho, tanto para os gestores públicos e privados quanto para as fontes pagadoras e os profissionais da área da saúde, além de configurar objeto de pesquisa para a comunidade científica. A prestação de serviços na área da saúde depende intensivamente de complexas interações entre pessoas, materiais, medicamentos, equipamentos e instalações, característica essa que eleva a chance de algo sair errado (Quinto, 2008).

Neste contexto em que a discussão sobre segurança do paciente ganha relevância, a qualidade assistencial torna-se um aspecto fundamental no monitoramento de indicadores de boas práticas clínicas (World Health Organization, 2009). Entretanto, a cultura da qualidade não está bem arraigada em parcela significativa das instituições de saúde no país, quer por desconhecimento, quer por falta de qualificação dos recursos humanos envolvidos, dentre outros aspectos.

Tendo em vista a necessidade de ações efetivas para o desenvolvimento de boas práticas na área da saúde, foi determinante a publicação do decreto n. 7.237, de 20 de julho de 2010, que regulamenta a lei n. 12.101, de 27 de novembro de 2009, e que dispõe sobre o processo de certificação das entidades beneficentes de assistência social para obtenção da isenção das contribuições para a seguridade social, uma vez que a isenção fiscal proveniente das instituições certificadas é aplicada em projetos diretamente ligados à rede pública.

Nesse contexto, o Ministério da Saúde mantém um Programa de Apoio ao Desenvolvimento Institucional do Sistema Único de Saúde (PROADI-SUS), com o objetivo geral de contribuir para o fortalecimento do SUS (Brasil, 2011).

Uma das possibilidades previstas na legislação surge com os hospitais de excelência, que, por suas expertise e qualificação na área de saúde, podem contribuir para o SUS de maneira diferenciada, não apenas na prestação de serviços assistenciais, mas também com uma participação mais consistente e sinérgica com os poderes públicos no desenvolvimento e fortalecimento do SUS (Brasil, 2011).

No capítulo i, artigos $1^{\circ}$ e $2^{\circ}$ da portaria 936, de 27 de abril, de 2011, está definido que os requisitos necessários para que as instituições sejam reconhecidas como hospitais de excelência são: instituição hospitalar filantrópica e com certificado de acreditação; planejamento e execução de projetos de apoio ao desenvolvimento institucional do SUS, nas quatro áreas: I - estudos de avaliação e incorporação de tecnologias, II - capacitação de recursos humanos, III - pesquisas de interesse público em saúde, IV - desenvolvimento de técnicas e operações de gestão em serviços de saúde; aplicação 
do valor das isenções fiscais com o percentual mínimo de 70\% em projetos, que são pactuados com os gestores públicos e avalizados pelo Ministério da Saúde, e 30\% em assistência direta; e os projetos desenvolvidos em parceria com as três esferas de governo devem ter duração máxima de três anos (Brasil, 2011).

O PROADI-SUS é caracterizado pelo estabelecimento de um vínculo formal entre seis hospitais de excelência e o Ministério da Saúde, mediante assinatura de termos de ajustes, de forma que venham a se refletir diretamente na melhoria do SUS (Ramos Filho, 2011). Cinco desses hospitais estão localizados no estado de São Paulo - Hospital Samaritano, Hospital Sírio-Libanês, Hospital Israelita Albert Einstein, Hospital Alemão Oswaldo Cruz e Hospital do Coração - e um, no Rio Grande do Sul - Hospital Moinhos de Vento.

Nesse contexto, emergiu o Programa de Intercâmbio Institucional Hospitais de Excelência e Hospitais Tutelados para o Desenvolvimento de Competências Técnicas na Área Assistencial a Pacientes Críticos, desenvolvido por um hospital privado filantrópico de médio porte, da cidade de São Paulo, reconhecido como hospital de excelência pelo Ministério da Saúde. O hospital está vinculado ao Ministério da Saúde no PROADI-SUS, como um projeto da área de responsabilidade social inserido nas áreas II e IV, correspondentes, respectivamente, à capacitação de recursos humanos e ao desenvolvimento de técnicas e operações de gestão em serviços de saúde.

O projeto foi realizado de novembro de 2008 a novembro de 2011 e atendeu 13 instituições participantes, selecionadas pelo Ministério da Saúde. Trata-se de uma experiência inovadora e incipiente em nosso meio, o que motivou a realização do presente estudo, com vistas a contribuir para a reflexão sobre as possibilidades de realização e de implementação de programas semelhantes pelo território nacional. As diferenças sociais e de recursos humanos e financeiros sabidamente são mais gritantes em regiões mais distantes do eixo Sul-Sudeste do país, razão pela qual esforços públicos e privados necessitam ser endereçados para essas regiões.

\section{Objetivos}

O presente estudo objetivou avaliar os padrões de qualidade existentes em unidades de terapia intensiva pediátrica e neonatal de hospitais públicos das regiões Norte, Nordeste e Centro-Oeste no que tange aos aspectos relacionados à estrutura e processos, nos períodos pré (diagnóstico situacional) e pós (reavaliação) desenvolvimento do programa educacional, descrever o programa educacional elaborado com base no diagnóstico situacional e apresentar os índices de capacitação e avaliação desse programa. 


\section{Metodologia}

Trata-se de um estudo descritivo, exploratório, 7 realizado em 13 hospitais da rede pública das regiões Norte, Nordeste e Centro-Oeste do Brasil. Os dados deste estudo correspondem aos coletados no período de novembro de 2008 a novembro de 2011. O programa foi desenvolvido em três fases.

A primeira fase, denominada diagnóstica, foi realizada por profissionais da área médica do hospital de excelência com o objetivo de conhecer cada instituição beneficiária. Foi elaborado um instrumento de avaliação tendo como base a portaria n. 3.432, de 12 de agosto de 1998, que estabelece o regulamento técnico para o funcionamento dos serviços de tratamento intensivo.

Ao pensarmos em padrão de qualidade, utilizamos o referencial de Donabedian (1988), que propõe um modelo unificado de avaliação de qualidade em saúde baseado em três componentes: 1) estrutura: refere-se aos atributos do ambiente em que o cuidado acontece, como estrutura física, recursos humanos, recursos materiais, recursos financeiros, entre outros; 2) processo: diz respeito à inter-relação entre o prestador e o receptor do cuidado, ou seja, é a maneira como o cuidado está implementado; 3) resultado: é o produto final da assistência prestada, considerando-se a saúde e a satisfação de padrões e de expectativas, ou seja, é a mudança do estado de saúde do paciente que pode ser atribuída a esse cuidado (Donabedian, 1988).

O instrumento elaborado para a avaliação diagnóstica das UTIs contemplou dois componentes: estrutura e processo. Para tanto, foram desenvolvidos dez critérios de avaliação; cada um deles contemplou elementos de mensuração, posteriormente tabulados para identificação dos índices de conformidade de cada critério. Os dez critérios de avaliação definidos no instrumento de coleta de dados foram os seguintes:

1) Recursos materiais e equipamentos: recursos tecnológicos adequados à unidade. 2) Recursos de estrutura hospitalar: estrutura física mínima e instalações. 3) Recursos assistenciais: serviços especializados para atender minimamente à UTI (serviços de diagnóstico e agência transfusional). 4) Recursos humanos/composição mínima da equipe: dimensionamento mínimo da equipe profissional da UTI e suas respectivas especializações. 5) Recursos humanos/avaliações médicas: avaliações realizadas por médicos especialistas. 6) Processos de atenção: protocolos estabelecidos, integração dos serviços hospitalares, reunião multiprofissional, educação permanente, sistematização da assistência de enfermagem, tracer (auditoria de prontuário), cursos de emergência (reanimação neonatal e Pediatric Advanced Life Support - PALS). 7) Processos de gestão: reavaliações periódicas de protocolos, normas e rotinas, da atribuição de cada profissional, dos indicadores assistenciais (escores prognósticos, grau de dependência, absenteísmo, turnover, taxa de ocupação, despesas, resultados, satisfação do usuário, 
relatórios multiprofissionais) e relatórios de gestão. 8) Política de humanização: controle do ambiente (luz, ruído, luz natural, temperatura), pais na unidade de terapia intensiva, visitas, método canguru, grupo de trabalho para a humanização em Utin. 9) Relação com o ambiente intra: interface com unidades de atenção à gestante, ao neonato e à criança criticamente enferma (centro cirúrgico, unidade neonatal, alojamento conjunto), critérios de internação e de alta bem estabelecidos (médicos e diretoria cientes), resultados técnicos e operacionais compartilhados com a diretoria da instituição. 10) Relação com o ambiente extra-hospitalar: interface com unidades de atenção extra-hospitalar à gestante, ao neonato e à criança gravemente enferma (outros hospitais, centrais de regulação de leitos, Serviço de Atendimento Móvel de Urgência - Samu), resultados técnicos e operacionais debatidos periodicamente com os gestores do SUS, critérios de internação e alta discutidos com os gestores do SUS.

Cada critério, com seus respectivos elementos de mensuração, foi avaliado mediante a classificação 'atende', 'atende parcialmente' e 'não atende'. Ao final, os critérios foram tabulados e resultaram em uma porcentagem de conformidade para cada item avaliado. Foi determinado um corte de $40 \%$ de conformidade a fim de melhor visualizar a realidade disposta em cada critério para o estabelecimento de prioridades de atenção e subsidiar a elaboração do programa educacional de recursos humanos. Essa porcentagem foi arbitrada pelos autores tendo em vista a inexistência de referencial semelhante na literatura nacional, o fato de ser um primeiro levantamento diagnóstico nas instituições públicas participantes do estudo e a experiência dos mesmos como avaliadores de qualidade em processos de acreditação.

A segunda fase teve início em setembro de 2010 e término em outubro de 2011, e foi constituída pelo programa educacional elaborado pelos autores do estudo. O referencial adotado baseou-se na pedagogia das competências e no construtivismo. Pautou-se, ainda, nas proposições do relatório da Comissão Internacional da Organização das Nações Unidas para a Organização das Nações Unidas para a Educação, a Ciência e a Cultura (Unesco), conhecido como Relatório Jacques Delors, que apresenta como pilares do conhecimento o aprender a conhecer, aprender a fazer, aprender a conviver e aprender a ser (Delors, 1998).

A terceira fase do programa, denominada reavaliação pós-programa educacional, foi realizada, em média, três meses após o término da capacitação em cada instituição beneficiária. $\mathrm{O}$ instrumento elaborado na primeira fase foi reaplicado a fim de se obterem os dados da reavaliação. Também foi utilizada a mesma metodologia para fins de comparabilidade. Essa fase teve início em fevereiro de 2011 e término em outubro de 2011.

Os índices do programa educacional foram obtidos por meio do total de horas/homem, calculado com base na carga horária do treinamento multiplicada 
pelo número de participantes. A média de horas/homem foi calculada pelo total de horas/homem treinado dividido pelo número médio de colaboradores treinados por instituição por mês (Garcia, Laurindo e Bittencourt, 2008).

Além disso, foi desenvolvida uma avaliação de reação para ser aplicada ao término de cada capacitação, com seis questões fechadas, cinco delas, voltadas ao conteúdo ministrado, classificadas em 'pouco relevante', 'nem muito nem pouco relevante', 'relevante' e 'muito relevante', e uma questão específica, relacionada aos facilitadores que conduziram a capacitação, classificada em 'inadequado para o tema', 'regular', 'bom', 'ótimo', 'excelente'. A última questão da avaliação foi aberta a fim de estimular os participantes a apresentarem seus comentários e sugestões.

Foi realizada análise descritiva dos dados, e os resultados foram apresentados em frequências absolutas e percentuais, médias e variações.

O projeto foi submetido e aprovado pelo Comitê de Ética em Pesquisa do Hospital Samaritano (São Paulo).

\section{Resultados e discussão}

Participaram do estudo 13 instituições (hospitais tutelados) em dez estados, a saber: Pará (PA), Maranhão (MA), Amapá (AP), Roraima (RR), Rondônia (RO), Piauí (PI), Goiás (GO), Tocantins (TO), Rio Grande do Norte (RN) e Alagoas (AL).

Gráfico 1

Distribuição dos índices de conformidade dos hospitais tutelados observados no pré (2009) e pós (2011) desenvolvimento do programa educacional: regiões Norte, Nordeste e Centro-Oeste do Brasil, 2009 e 2011.

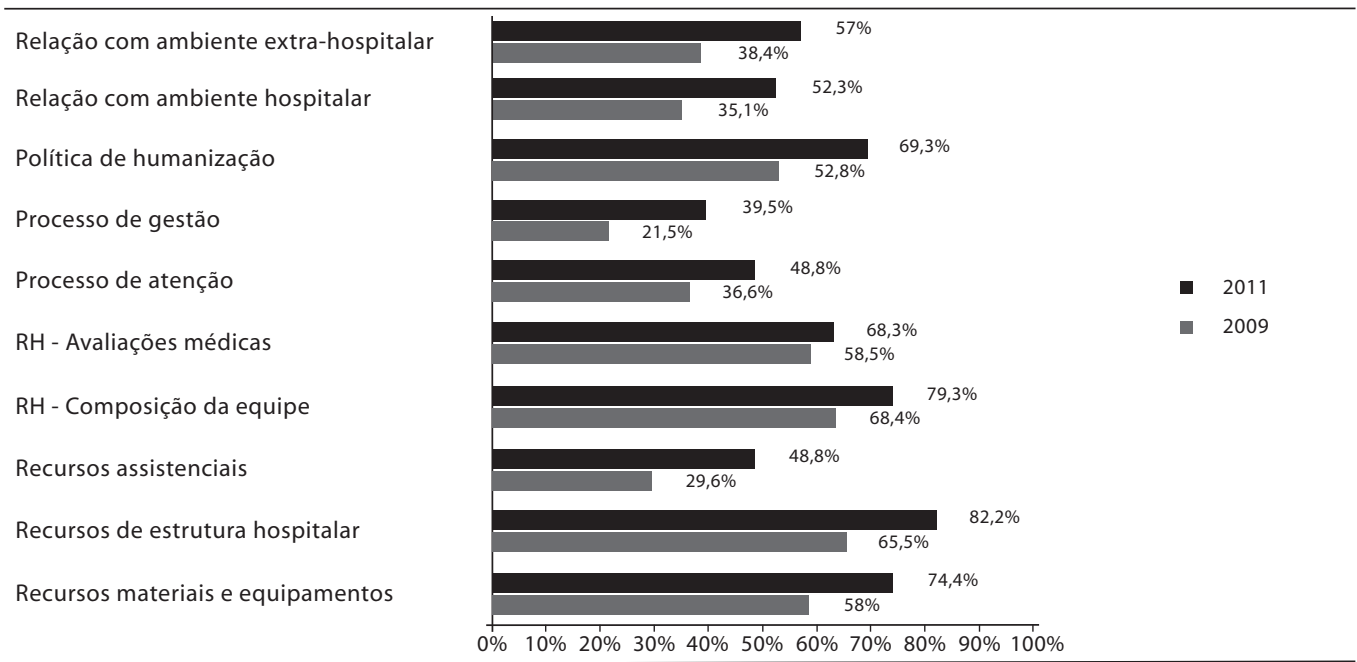

Fonte: Os autores. 
No diagnóstico inicial (2009), conforme o gráfico 1, foi constatado que cinco critérios de avaliação se encontravam abaixo de $40 \%$ de conformidade: recursos assistenciais, processos de gestão, processos de atenção, relação com o ambiente hospitalar e relação com o ambiente extra-hospitalar.

Os recursos assistenciais referem-se aos serviços de apoio e diagnóstico para atender minimamente às necessidades das UTIs. A maioria dos hospitais era provida de aparelhos de raios-X; entretanto, não dispunham de ultrassonografia e ecocardiografia à beira do leito, cuja necessidade está prevista na portaria n. 3.432/1998. A maioria dos serviços de apoio e diagnóstico era terceirizada, e muitas vezes não havia atendimento emergencial, apenas com agendamento - por exemplo, para procedimentos de tomografia computadorizada, ressonância nuclear magnética, endoscopia, serviço de hemodinâmica, entre outros. Em todos os serviços, foi evidenciada a presença de agência transfusional nas 24 horas.

Os resultados de processos de gestão e atenção refletiam a necessidade das instituições de disporem de normas, rotinas, protocolos e indicadores assistenciais e administrativos formalizados, para subsidiar suas atividades e justificar suas necessidades.

A relação com o ambiente hospitalar pode ser compreendida como parte do processo de gestão hospitalar, uma vez que a interface com as unidades intra-hospitalares deve permear toda a equipe profissional, com normas, rotinas e indicadores bem estabelecidos e divulgados. Já a relação com o ambiente extra-hospitalar não depende apenas do gestor hospitalar, mas da política de atenção básica à saúde para a população em discussão, assim como da estrutura oferecida para esse atendimento. Em uma das instituições (Alagoas) foi evidenciado um transporte específico para recém-nascidos denominado Serviço de Atendimento Móvel de Urgência Neonatal (Samu Neo), o qual, no entanto, ainda é subutilizado.

Outro aspecto de relevância no diagnóstico das UTIs foi a variação dos índices de cada critério avaliado nas 13 instituições. Alguns critérios apresentaram grandes variações, mesmo aqueles com conformidade superior a 40\%. Observou-se que nos recursos humanos, tanto em relação à composição mínima da equipe quanto nas avaliações de especialistas médicos, o índice de conformidade variou de 10 a $100 \%$. No critério recursos materiais e equipamentos, observou-se uma média de conformidade de $58 \%$; contudo, a variação entre os índices oscilou de 21,7 a 92\%. Tal fato comprova o crescimento das UTIs sem adequada estrutura física e recursos tecnológicos necessários para o mínimo funcionamento, como previsto em lei. Outra variação importante nos resultados foi observada em relação à política de humanização, com índices que variaram entre 9,1 a 90\% de conformidade.

Pesquisa realizada pela Associação de Medicina Intensiva Brasileira (Amib) corrobora o diagnóstico situacional deste estudo acerca do cenário 
das UTIs do país, incluindo as UTIs para adultos. De maneira geral, também confirma a distribuição desigual do número de leitos de UTI, com maior concentração nas regiões Sul, Sudeste e Centro-Oeste. É necessário estimar de 1 a 3 leitos de UTI para cada 10 mil habitantes, no entanto, em números, a distribuição ocorre da seguinte forma: na região Sul há 1,6 leitos para 10 mil habitantes, na região Sudeste há 1,7 leitos, na região Centro-Oeste há 1,4 leitos e nas regiões Nordeste e Norte há 0,8 leitos para cada 10 mil habitantes (Associação de Medicina Intensiva Brasileira, 2008).

No presente estudo, foi observada superlotação das Utins e Utips. Além disso, a regulação de leitos parece não ser efetiva em muitas das instituições e o transporte (ambulância) se mostrou inadequado para neonatos, crianças graves e gestantes, com exceção do referido serviço em Maceió. Os dados refletiram, ainda, a desigualdade da distribuição de mão de obra especializada para essas regiões, o que reforça a necessidade de desenvolvimento de programas de capacitação específicos para esses profissionais. Na tentativa de contribuir com o quantitativo e o qualitativo de profissionais especializados nas regiões Norte, Nordeste e Centro-Oeste do país, em novembro de 2010 foram implantados sessenta novos programas de residência médica em especialidades prioritárias para o SUS (Brasil, 2010). Todavia, ainda são poucas as iniciativas como essa para as demais categorias profissionais.

Outro fato relevante é que em algumas unidades, principalmente em Utips, foi observada a não permissão de acompanhantes, sendo que os pais eram considerados visitantes e, portanto, submetidos aos horários regulares de visita. Atualmente, sobretudo em Utins e Utips, é impossível discutir a assistência sem a inclusão da família. Dentro da UTI, a família pode participar de todo o processo terapêutico. Em Utins, por exemplo, nas quais o período de permanência de um prematuro, por exemplo, muitas vezes é prolongado, os pais podem ser requisitados para permanecer na unidade em função do método canguru (nas três fases), que contribui positivamente na evolução clínica do neonato. Diversos estudos investigam e apontam para a importância da permanência da família na UTI. Além disso, o Estatuto da Criança e do Adolescente (Brasil, 2008) garante que crianças de 0 a 18 anos de idade usufruam o direito de um acompanhante durante sua internação, independentemente da unidade (Ferreira, Sakita e Ceccon, 2009; Molina et al., 2007). Na reavaliação foi possível observar que a totalidade das instituições dispunha do acompanhamento dos pais dentro das UTIs; no entanto, duas instituições estavam em fase de adaptação a essa rotina, e os pais permaneciam por um período determinado, tendendo a prorrogação.

Ainda que as melhorias observadas não possam ser atribuídas exclusivamente ao programa educacional realizado e às ações correlatas ao projeto como um todo, foi observado um aumento nos índices dos dez critérios avaliados nas 13 instituições beneficiárias. Apenas o critério 'Processo de 
gestão' permaneceu com índice inferior a 40\%, o que reflete a necessidade de monitoramento e avaliação periódica dos processos gerenciais implantados nas instituições. Os critérios 'Relação com o ambiente intra-hospitalar' e 'Relação com o ambiente extra-hospitalar' foram os que apresentaram índices de melhoria para um menor número de instituições (38\% e $46 \%$ das instituições, respectivamente).

Para todos os critérios analisados, a média de melhoria observada foi de $15,2 \%$ com variação de $9,8 \%$ a $19,2 \%$. Todavia, ainda que as melhorias tenham sido identificadas, o instrumento de avaliação não foi sensível suficiente para identificar as melhorias qualitativas, o que caracteriza uma limitação deste estudo.

Os fatores observados pelos autores no decorrer do projeto, quer sejam decorrentes das necessidades indicadas pelo diagnóstico efetuado, pelo programa educacional ou por ações do Ministério da Saúde que contribuíram para a melhoria de cada critério, foram:

1) Recursos materiais e equipamentos (aumento de 16,4\%): o Ministério da Saúde disponibilizou equipamentos específicos ao atendimento à criança de alto risco para $76 \%$ das instituições beneficiárias. Já os materiais de uso contínuo são adquiridos pela própria instituição, por meio de licitações.

2) Recursos de estrutura hospitalar (aumento de 16,7\%): uma instituição teve a oportunidade de reconstruir toda a área de Utin, com a adequação total das suas instalações. Nas demais instituições, foram observadas melhorias pontuais relativas à estrutura interna da UTI e à reorganização de salas e de serviços.

3) Recursos assistenciais (aumento de 19,2\%): 76\% das instituições apresentaram melhorias nos serviços diagnósticos, uma das instituições, inclusive, adquiriu tomógrafo próprio.

4) Recursos humanos/Composição da equipe (aumento de 10,9\%): 56\% das instituições apresentaram aumento nos seus índices em razão de readequações quantitativas no dimensionamento do quadro de colaboradores.

5) Recursos humanos/Avaliações médicas (aumento de 9,8\%): 61,5\% das instituições apresentaram elevação nos seus índices como resultado da reorganização dos especialistas para atender as necessidades das crianças internadas em unidades críticas.

6) Processos de atenção (aumento de 12,2\%): 76\% das instituições apresentaram aumento nos seus índices, uma vez que fizeram seu mapeamento de processos e que estão em fase de discussão ou implantação de indicadores clínicos e administrativos e da sistematização da assistência de enfermagem. 7) Processos de gestão (aumento de 18\%): 84\% das instituições obtiveram melhoria em seus índices, por compreenderem a importância da revisão de seus processos, do acompanhamento dos seus indicadores e da discussão com a equipe a fim de propor outras melhorias. 
8) Política de humanização (aumento de 16,5\%): 84\% das instituições apresentaram elevação em seus índices por terem seguido as diretrizes do Estatuto da Criança e do Adolescente, além de terem melhorado as acomodações para os pais acompanhantes.

9) Relação com o ambiente intra-hospitalar (aumento de 16,7\%): 38\% das instituições obtiveram aumento dos índices em decorrência da compreensão da importância da interface com outras áreas intra-hospitalares, até mesmo para o compartilhamento de responsabilidades que impactam diretamente a assistência à criança criticamente enferma.

10) Relação com o ambiente extra-hospitalar (aumento de 16,4\%): 46\% das instituições apresentaram elevação em seus índices por terem oportunizado discussões com secretarias de saúde e outros órgãos a fim de propor melhorias no atendimento à sua demanda.

Os resultados que tratam da descrição do programa educacional e seus indicadores específicos são descritos a seguir. O programa educacional foi elaborado pela equipe técnica do projeto e seu conteúdo foi composto por treinamento on the job (realizado em cada instituição beneficiária) e curso com visita técnica monitorada no hospital de excelência em São Paulo, com carga horária total de 42 horas.

O treinamento on the job, com carga horária de 12 horas, foi realizado por duas enfermeiras de responsabilidade social à beira do leito e mediante reuniões com as equipes multiprofissionais, que englobaram os seguintes assuntos: sensibilização para a adequada avaliação de dor em neonatologia e pediatria (teoria e prática); aspectos da humanização em UTI (conforto do neonato, diminuição dos estressores ambientais, presença dos pais na UTI); tipos de curativos utilizados na cobertura de acessos venosos periféricos e centrais; diminuição do estoque de medicamentos nas UTIs (para diminuir o risco de problemas relacionados a medicamentos); checagem diária de planilhas consideradas fundamentais para a segurança do atendimento (carro de emergência, temperatura de geladeira, controle de materiais fixos do setor, controle de materiais encaminhados e retirados da central de materiais esterilizados, entre outros); avaliação dos registros de enfermagem; sistematização da assistência de enfermagem; e boas práticas assistenciais em geral. Vale ressaltar que o treinamento on the job variou em abordagem, de acordo com a realidade de cada instituição, e seus índices de participação são apresentados na tabela 1 . 


\begin{tabular}{|c|c|c|c|}
\hline Instituição & $\begin{array}{c}\text { N. de participantes na } \\
\text { apresentação do } \\
\text { programa }\end{array}$ & N. de temas abordados & $\begin{array}{l}\text { N. de colaboradores } \\
\text { (registrados) no } \\
\text { treinamento }\end{array}$ \\
\hline Hospital A (PA) & 20 & 3 & 25 \\
\hline Hospital B (AP) & 22 & 3 & 1 \\
\hline Hospital C (MA) & 21 & 6 & 41 \\
\hline Hospital D (MA) & 14 & 7 & 29 \\
\hline Hospital E (RN) & 22 & 6 & 8 \\
\hline Hospital F (AL) & 2 & 5 & 15 \\
\hline Hospital G (GO) & 5 & 6 & 13 \\
\hline Hospital H (GO) & 12 & 5 & 12 \\
\hline Hospital I (PI) & 12 & 6 & 49 \\
\hline Hospital J (TO) & 4 & 2 & 12 \\
\hline Hospital K (RR) & 25 & 7 & 42 \\
\hline Hospital L (RO) & 5 & 10 & 17 \\
\hline Hospital M (PA) & 20 & 5 & 26 \\
\hline Total & 184 & & 290 \\
\hline
\end{tabular}

Fonte: Os autores.

O treinamento on the job para as 13 instituições nas regiões do estudo totalizou 144 horas em que 290 participantes foram distribuídos de forma heterogênea, o que não possibilitou o cálculo clássico de horas/homem, uma vez que a participação dos profissionais de saúde foi flutuante. Entretanto, para a temática abordada acerca da sensibilização do profissional para o adequado manejo da dor neonatal e pediátrica, que representa um aspecto da capacitação on the job, foi possível estabelecer o cálculo de horas/homem, uma vez que a teoria foi desenvolvida em sala de aula com exposições dialogadas (duração de uma hora) e com público alvo limitado à sala. Nessa temática, então, foram capacitados 103 profissionais de saúde, com um total de 103 horas/homem de treinamento e média de 8 horas/homem, das seguintes categorias profissionais: enfermeiros $(43 \%)$, técnicos de enfermagem $(18 \%)$, médicos (18\%) e outras categorias (16\%). Dos 103 profissionais, $91(87 \%)$ responderam à questão referente ao tempo de formação, identificando-se que $50 \%$ estavam formados há oito anos ou mais, o que revela a necessidade de atualização dos conhecimentos.

Os participantes da capacitação consideraram: a relevância da temática da dor: muito relevante $(80 \%)$ e relevante $(20 \%)$; o método pedagógico 
utilizado: muito relevante (62\%) e relevante (38\%); a avaliação da capacitação de forma geral: muito relevante $(81 \%)$ e relevante $(19 \%)$; e os facilitadores: excelentes $(67 \%)$, ótimos $(29 \%)$ e bons (4\%). Os comentários adicionais foram apresentados por sessenta participantes (58\%). O agrupamento das respostas foi categorizado da seguinte forma: relacionadas à compreensão da relevância do tema abordado para a melhoria de ações na prática clínica (55\%); mencionando a continuidade da capacitação com programa de treinamento, com maior carga horária, incluindo treinamento on the job e educação continuada (32\%); e enfatizando a necessidade de maior número de profissionais participantes de treinamentos multiprofissionais sobre o tema $(13 \%)$.

Em relação à etapa do programa desenvolvida em São Paulo (curso com visita técnica monitorada), o enfoque foi direcionado para controle de infecção hospitalar, farmácia hospitalar, engenharia clínica, sistematização da assistência de enfermagem, gestão de pessoas e indicadores assistenciais e de gestão. A carga horária de 30 horas foi distribuída em visitas monitoradas, palestras e fóruns de discussão, e envolveu profissionais de diversas áreas da instituição (assistenciais, de apoio e de gestão), conforme demonstram os dados da tabela 2 .

Tabela 2

Indicadores referentes ao curso e à visita monitorada em 13 instituições beneficiárias do programa educacional: regiões Norte, Nordeste e Centro-Oeste do Brasil, 2010-2011.

\begin{tabular}{cccc}
\hline Instituição & $\begin{array}{c}\text { N. de representantes por } \\
\text { instituição }\end{array}$ & $\begin{array}{c}\text { N. de áreas envolvidas } \\
\text { direta e indiretamente na } \\
\text { semana de atividades }\end{array}$ & $\begin{array}{c}\text { N. de temas abordados } \\
\text { durante a semana }\end{array}$ \\
\hline Hospital A (PA) & 2 & 25 & 18 \\
Hospital B (AP) & 2 & 25 & 18 \\
Hospital C (MA) & 2 & 26 & 19 \\
Hospital D (MA) & 2 & 26 & 19 \\
Hospital E (RN) & 2 & 29 & 21 \\
Hospital F (AL) & 2 & 29 & 21 \\
Hospital G (GO) & 2 & 26 & 20 \\
Hospital H (GO) & 3 & 26 & 20 \\
Hospital I (PI) & 2 & 26 & 20 \\
Hospital J (TO) & 2 & 26 & 18 \\
Hospital K (RR) & 2 & 25 & 18 \\
Hospital L (RO) & 2 & 25 & 20 \\
Hospital M (PA) & 2 & 26 & \\
Total & 27 & & 20 \\
\hline
\end{tabular}

Fonte: Os autores. 
O curso e visitas monitoradas totalizaram 810 horas/homem de treinamento em São Paulo. A média de 180 horas/homem foi observada nessa etapa, uma vez que a variação foi de 2 a 6 participantes/mês (média de 4,5). Considerando a média de áreas do hospital de excelência envolvidas (26) nesses treinamentos (com seu consequente número de profissionais por área), observa-se a magnitude da estrutura disponibilizada para essa capacitação, visando à qualidade do programa. As aulas preparadas para serem ministradas presencialmente foram gravadas em DVD e destinadas às instituições participantes a fim de serem compartilhadas por todos os profissionais interessados e pelos serviços de educação permanente, quando existentes, de forma a facilitar a socialização dos conteúdos ministrados.

O programa foi constituído, ainda, de treinamentos de urgência e emergência em Pediatric Advanced Life Support (PALS) e em reanimação neonatal, para profissionais da pediatria e da neonatologia respectivamente, o que elevou a carga horária total do programa de 42 horas para 66 horas, com consequente aumento do número de horas/homem treinado.

O PALS, com carga horária de 16 horas, foi oferecido pelo período de cinco meses em cinco polos: Belém, São Luís, Natal, Porto Velho e Goiânia, totalizando 110 profissionais treinados. O número médio de profissionais treinados por mês foi 22, perfazendo, portanto, um total de 1.760 horas/homem treinados e uma média de 80 horas/homem de treinamento. A tabela 3 apresenta os dados referentes ao curso do PALS realizado nos cinco polos.

Tabela 3

Indicadores referentes ao curso PALS oferecido às instituições beneficiárias, realizado nos cinco polos: regiões Norte, Nordeste e Centro-Oeste do Brasil, 2010-2011.

\begin{tabular}{cccccccc}
\hline & Participantes & \multicolumn{2}{c}{ Aprovados } & \multicolumn{2}{c}{ Profissionais para } & \multicolumn{2}{c}{ Não aprovados } \\
Polo & $f$ & $f$ & $\%$ & refazer a prova teórica & $f$ & $\%$ \\
& & & & $f$ & $\%$ & & \\
\hline São Luís & 24 & 14 & 58 & 5 & 21 & 5 & 21 \\
Natal & 26 & 7 & 27 & 7 & 27 & 12 & 46 \\
Belém & 19 & 6 & 32 & 4 & 21 & 9 & 47 \\
Goiânia & 27 & 14 & 52 & 8 & 30 & 5 & 18 \\
Porto Velho & 14 & 10 & 72 & 2 & 14 & 2 & 14 \\
Total & 110 & 51 & 46,3 & 26 & 23,7 & 33 & 30 \\
\hline
\end{tabular}

Fonte: Os autores.

$f=$ frequência absoluta.

$\%=$ frequência percentual. 
O curso de reanimação neonatal, com carga horária de 8 horas, foi realizado em cada instituição beneficiária pelo período de dois meses, totalizando 257 profissionais treinados. O número médio de profissionais treinados no mês foi de 128,5, obtendo-se um total de 2.056 horas/homem treinados e uma média de 16 horas/homem treinados. A tabela 4 apresenta os dados referentes ao curso de reanimação neonatal realizado em cada instituição beneficiária em parceria com a Sociedade Brasileira de Pediatria.

Tabela 4

Indicadores referentes ao curso de reanimação neonatal oferecido às instituições beneficiárias: regiões Norte, Nordeste e Centro-Oeste do Brasil, 2010-2011.

\begin{tabular}{cccccc}
\hline & & \multicolumn{2}{c}{ Participantes } & \multicolumn{2}{c}{ Aprovados } \\
Estado & N. de inscritos & $f$ & $\%$ & $f$ & $\%$ \\
& & & & & \\
\hline Maranhão & 40 & 40 & 100 & 38 & 95 \\
Pará & 40 & 37 & 92,5 & 37 & 100 \\
Goiás & 40 & 27 & 67,5 & 27 & 100 \\
Tocantins & 20 & 19 & 95 & 19 & 100 \\
Piauí & 20 & 20 & 100 & 15 & 75 \\
Rio Grande do Norte & 28 & 25 & 89,2 & 25 & 100 \\
Alagoas & 22 & 20 & 91 & 20 & 100 \\
Rondônia & 22 & 22 & 100 & 21 & 95 \\
Roraima & 20 & 20 & 100 & 19 & 95 \\
Amapá & 26 & 25 & 96 & 21 & 84 \\
Total & 278 & 255 & 92 & 242 & 95 \\
\hline
\end{tabular}

Fonte: Os autores.

$f=$ frequência absoluta.

$\%=$ frequência percentual.

Por se tratar de um programa filantrópico, todo o conteúdo foi oferecido sem qualquer tipo de ônus financeiro para os profissionais participantes.

Quanto aos indicadores de treinamento evidenciados no programa, a comparação com a literatura é muito limitada, tendo em vista a escassez de publicações que os contemplem. Os poucos existentes e descritos se relacionam aos treinamentos admissionais e não podem ser comparados com os obtidos em programas específicos ou inéditos, como o do presente estudo. A carga horária dos treinamentos admissionais para alguns autores tem variado de 42 a 60 horas (Cunha e Valério, 2009; Garcia, 2011) ou contemplam 24 horas de treinamento quando voltados para equipe multiprofissional 
(Garcia, 2011). O treinamento admissional tem como objetivo alinhar o conhecimento entre os profissionais sobre rotinas, procedimentos e protocolos, bem como promover a sua integração na equipe e no contexto institucional (Cunha e Valério, 2009).

Este programa educacional buscou o alinhamento à abordagem da educação permanente em saúde, que prevê a educação no trabalho centrada em treinamentos e atualizações técnicas, em especial propostas que busquem aproximar educação e trabalho, concebidos, ambos, como práticas sociais (Montanha e Peduzzi, 2010).

Como apontado por outros autores, trabalhar a educação permanente em saúde possibilita uma reflexão dos profissionais em relação ao fazer e ao pensar como está sendo feito, abrindo oportunidades para o diálogo entre os diversos saberes, assim como para a construção de um conhecimento e de uma inteligência crítica e coletiva (Rodrigues, Vieira e Torres, 2010).

Mais do que alinhar institucionalmente, posto que contempla diversas instituições, o programa objetivou qualificar os recursos humanos para temas prioritários na atenção à saúde e que reforcem a qualidade e a segurança das práticas assistenciais, incluindo, ainda, a gestão dos processos administrativos e assistenciais. A proposta buscou, assim, um nivelamento dentro do SUS, de forma a construir um arcabouço comum para maior aprofundamento e evolução em programas posteriores, ou mesmo para a ampliação da autonomia desses serviços.

\section{Conclusões}

A metodologia adotada para a realização do diagnóstico das 13 instituições das regiões Norte, Nordeste e Centro-Oeste forneceu dados para o conhecimento da estrutura e dos processos desenvolvidos. Foi observada a diversidade de recursos entre as várias regiões, bem como a elevada variação nos índices de conformidade dos critérios avaliados. Embora tenha constituído um desafio para o planejamento da capacitação dos profissionais, os resultados mostram um índice de horas/homem de treinamento que pode ser considerado satisfatório por ser mais elevado quando comparado aos índices descritos na literatura, ainda que não específicos para esse tipo de capacitação, tendo em vista que parcela significativa das instituições não dispõe de educação permanente ativa.

A participação dos profissionais de diversas áreas do hospital de excelência em um programa de responsabilidade social tem sido relevante para os resultados alcançados até o momento e apresentados neste estudo, o que revela os benefícios das parcerias público-privadas em nosso meio. A aproximação de profissionais do hospital de excelência com os profissionais 
participantes do programa proporcionou um espaço de discussão e troca de experiências, cujo objetivo maior foi a busca de práticas seguras com foco no resultado, respeitando a realidade de cada região e de cada instituição partícipe. Essa estratégia, além de possibilitar a atualização técnica dos profissionais participantes do programa, permitiu a reflexão e a análise crítica dos processos de trabalho, facilitando a identificação de problemas e a elaboração de planos de ação para a superação dos mesmos.

Foi possível verificar também que o programa contribuiu para o aumento dos índices de conformidade em relação à maioria dos critérios avaliados, resultado positivo e expressivo para um programa inédito e inovador no Brasil, considerando a complexidade e peculiaridades do setor público de saúde e as diferenças regionais existentes.

O Ministério da Saúde avaliou positivamente o projeto, na medida em que a sua abrangência atendeu aos princípios de universalidade, equidade e integralidade do SUS e estava em acordo com as políticas e diretrizes de saúde em âmbito federal. Além disso, o programa contribuiu com a melhoria da assistência prestada no âmbito das UTIs neonatais e pediátricas (evidenciada na fase de reavaliação) e, portanto, aprovou a continuidade e ampliação do programa, cuja proposta principal é o monitoramento de indicadores assistenciais e administrativos, para o próximo triênio (2012-2014).

\section{Colaboradores}

Os autores declaram que participaram da concepção e do planejamento do projeto de pesquisa, obtenção ou análise e interpretação dos dados, redação e revisão crítica do manuscrito.

\section{Notas}

\footnotetext{
1 Hospital Samaritano de São Paulo, São Paulo, SP, Brasil.

Doutoranda pela Escola Paulista de Enfermagem da Universidade Federal de São Paulo. <edna.bussotti@samaritano.org.br>

Correspondência: Rua Ernesto dos Santos, 247, Jardim Independência, CEP 03225-000, São Paulo, São Paulo, Brasil.
} 


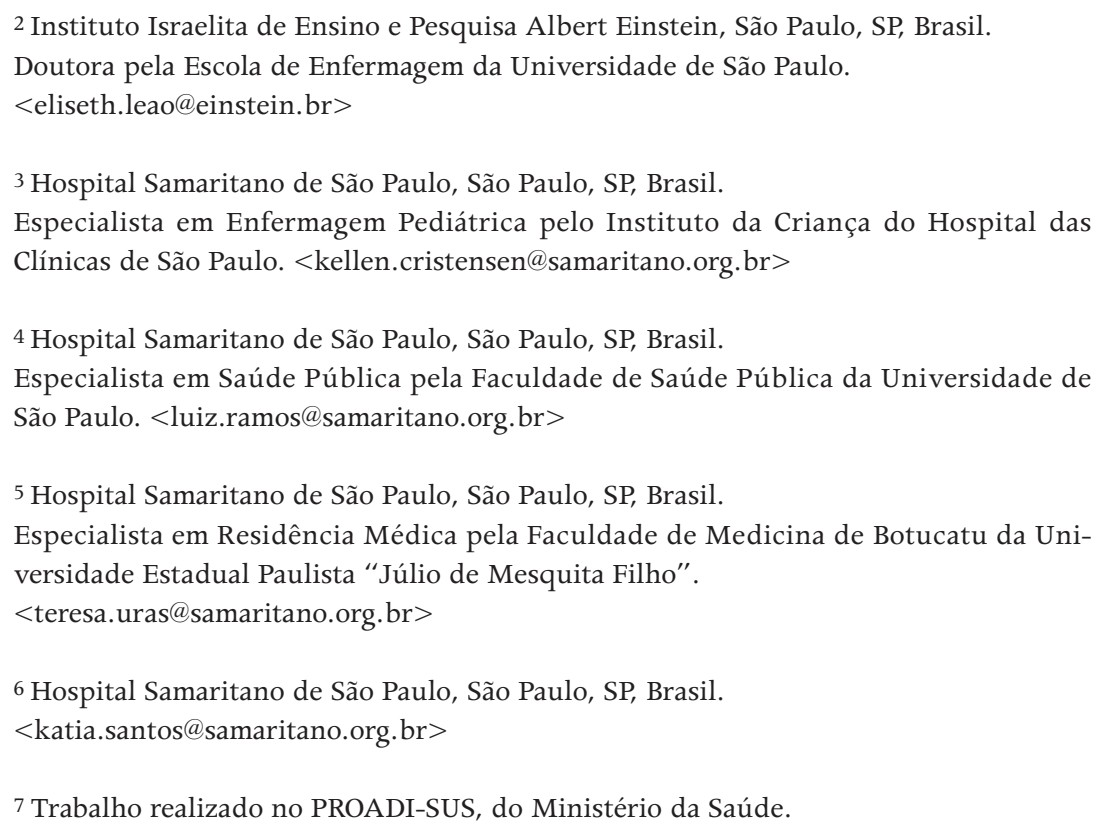

\section{Referências}

ABREU, Daisy M. X.; CÉSAR, Cibele C.; FRANÇA, Elisabeth B. Relação entre as causas de morte evitáveis por atenção à saúde e a implementação do Sistema Único de Saúde no Brasil. Revista Panamericana de Salud Pública, Washington, v. 21, n. 5, p. 282-289, 2007.

ALMEIDA, Maria F. B.; GUINSBURG, Ruth. Programa de reanimação neonatal da sociedade brasileira de pediatria: condutas 2011. Disponível em: <www.sbp.com.br/ pdfs/PRN-SBP-ReanimaçãoNeonatalFinal2011-25mar11.pdf>. Acesso em: 10 jun. 2011.

ASSOCIAÇÃO DE MEDICINA INTENSIVA BRASILEIRA (AMIB). Censo AMIB, 2008. Disponível em: <www.amib.org.br>. Acesso em: 27 jan. 2011.
BARBOSA, Arnaldo P. Terapia intensiva neonatal e pediátrica no Brasil: o ideal, o real e o possível. Jornal de Pediatria, Rio de Janeiro, v. 80, n. 6, p. 437-438, 2004.

BRASIL. Ministério da Educação. Programas de residência médica beneficiarão regiões carentes. 2 dez. 2010. Disponível em: <http:// portal.mec.gov.br>. Acesso em: 26 jan. 2011.

Ministério da Saúde. Agência Nacional de Vigilância Sanitária. RDC n. 50, de 21 de fevereiro de 2002. Dispõe sobre o Regulamento Técnico para planejamento, programação, elaboração e avaliação de projetos físicos de estabelecimentos assistenciais de saúde. Disponível em: <www.anvisa.gov. br/legis/resol/2002/50_02rdc.pdf>. Acesso em: 20 dez. 2012. 
. Ministério da Saúde. Portaria n. 3.432, de 12 de agosto de 1998. Estabelece Critérios de Classificação de Unidade de Terapia Intensiva (UTI). Diário Oficial da União n. 154; Poder Executivo, 13 ago. 1998. Disponível em: <www.medicinaintensiva. com.br/portaria-3432.htm>. Acesso em: 10 jan. 2010.

Ministério da Saúde. Portaria n. 1.996, de 20 de agosto de 2007. Dispõe sobre as diretrizes para a implementação da Política Nacional de Educação Permanente em Saúde e dá outras providências. Brasília: Ministério da Saúde, 2007. Disponível em: $<$ http://bvsms.saude.gov.br/bvs/publicacoes/ politica_nacional_educacao_permanente_ saude.pdf>. Acesso em: 28 dez. 2011.

Ministério da Saúde. Sistema de Informação sobre Mortalidade (SIM): painel de monitoramento da mortalidade infantil e neonatal. Disponível em: < http://svs.aids. gov.br/dashboard/mortalidade/infantil.show. mtw>. Acesso em: 6 jun. 2011.

Ministério da Saúde. Lei n. 12.101, de 27 de novembro de 2009. Dispõe sobre a certificação das entidades beneficentes de assistência social; regula os procedimentos de isenção de contribuições para a seguridade social; altera a Lei n. 8.742, de 7 de dezembro de 1993; revoga dispositivos das Leis nos 8.212, de 24 de julho de 1991, 9.429, de 26 de dezembro de 1996, 9.732, de 11 de dezembro de 1998, 10.684, de 30 de maio de 2003, e da Medida Provisória n. 2.187-13, de 24 de agosto de 2001; e dá outras providências. Diário Oficial da União, Brasília, 30 de nov. 2009. Disponível em: $<$ http://legislacao.planalto.gov.br/legisla/ legislacao.nsf/Viw_Identificacao/lei\%2012. 101-2009? OpenDocument>. Acesso em: 20 dez. 2011.

Ministério da Saúde. Decreto n. 7.237, de 20 de julho, de 2010. Regulamenta a lei n. 12.101, de 27 de Novembro de 2009, para dispor sobre o processo de certificação das entidades beneficentes de assistência social para obtenção da isenção das contribuições para a seguridade social, e dá outras providencias. Diário Oficial da União, Brasília, 21 jul. 2010. Disponível em: <http:// legislacao.planalto.gov.br/legisla/legslacao. nsf/Viw_Identificacao/DEC\%207.237-2010? OpenDocument>. Acesso em: $11 \mathrm{dez} .2011$.

Ministério da Saúde. Lei n. 936, de 27 de abril de 2011. Dispõe sobre as regras e critérios para apresentação, monitoramento, acompanhamento e avaliação de projetos do programa de apoio ao desenvolvimento do Sistema Único de Saúde (PROADI/SUS). Brasília, DF, 28 de abril de 2011. Seção 1, n. 80. Disponível em: <http://portal.saude.gov.br/ portal/arquivos/pdf/nota_hosp_excelencia. pdf $>$. Acesso em: 27 jun. 2011.

Ministério da Saúde. Secretaria de Atenção à Saúde. Lei n. 8.069, de 13 de julho de 1990. Estatuto da criança e do adolescente. 3. ed. Brasília: Ministério da Saúde, 2008. Capítulo I: Do direito à vida e à saúde.

CECCIM, Ricardo B.; FEUERWERKER, Laura C. M. O quadrilátero da formação para a área da saúde: ensino, gestão, atenção e controle social. Physis: Revista de Saúde Coletiva, Rio de Janeiro, v. 14, n. 1, p. 41-65, 2004.

CUNHA, Lissandra B.; VALÉRIO, Selma T. Treinamento admissional. Einstein: educação continuada em saúde, São Paulo, v. 7, n. 1, p. 49-51, 2009.

DELORS, Jacques. Educação: um tesouro a descobrir. São Paulo: Cortez, 1998.

DONABEDIAN, Avedis. The Quality of Care: Who Can it Be Assessed?. JAMA, v. 260, n. 12, p. 1743-1748, 1988.

FERREIRA, Juliana C. O. A.; SAKITA, Neusa K.; CECCON, Maria E. J. R. Experiência de grupo de pais em uma unidade de terapia intensiva neonatal. Pediatria (São Paulo), São Paulo, v. 31, n. 1, p. 20-25, 2009.

FRANÇA, Elisabeth; LANSKY, Sônia. Mortalidade infantil neonatal no Brasil: situação, tendências e perspectivas. Rede Interagencial de Informação para a Saúde Demografia e saúde: contribuição para análise de 
situação e tendências. Brasília: Opas, 2009. p. 83-112.

GARCIA, Simone C. Treinamento do recém-admitido. Entrevista ao Portal da Enfermagem. 6 Jan. 2011. Disponível em: <www. portaldaenfermagem.com.br/entrevistas_ read.asp?id=62>. Acesso em: 28 jul. 2011.

GARCIA, Simone C.; LAURINO, Patrícia S.; BITTENCOURT, Claudia M. Indicadores de treinamento. In: LEÃO, Eliseth R. et al. (Orgs.). Qualidade em saúde e indicadores como ferramenta de gestão. São Paulo: Yendis, 2008. p. 75-86.

MALTA, Deborah C. et al. Lista de causas de mortes evitáveis por intervenções do Sistema Único de Saúde do Brasil. Epidemiologia em Serviços de Saúde, Brasília, v. 16, n. 4, p. 233-244, out. /dez. 2007.

MOLINA, Rosemeire C. M. et al. Presença da família nas unidades de terapia intensiva pediátrica e neonatal: visão da equipe multidisciplinar. Revista de Enfermagem da Escola Anna Nery, Rio de Janeiro, v. 11, n. 3, p. 437-442, set. 2007.

MONTANHA, Dionize; PEDUZZI, Marina. Educação permanente em enfermagem: levantamento de necessidades e resultados esperados segundo a concepção dos trabalhadores. Revista da Escola de Enfermagem da Universidade de São Paulo, São Paulo, v. 44, n. 3, p. 597-604, 2010.

NEPOMUCENO, Lilian M. R.; KURCGANT, Paulina. Uso de indicador de qualidade para fundamentar programa de capacitação de profissionais de enfermagem. Revista da Escola de Enfermagem da Universidade de São Paulo, São Paulo, v. 42, n. 4, p. 665-672, 2008.
QUINTO, Antônio N. A responsabilidade corporativa dos gestores de organizações de saúde e a segurança do paciente. Revista de Administração em Saúde, São Paulo, v. 10, n. 41, p. $140-6,2008$.

RAMOS FILHO, Luiz M. Entidades filantrópicas de reconhecida excelência. O caso do Hospital Samaritano. A relação com o SUS e perspectivas. In: MODESTO, Paulo; CUNHA JUNIOR, Luiz Arnaldo. Terceiro Setor e parcerias na área de saúde. Belo Horizonte: Fórum, 2011. p. 207-208.

RODRIGUES, Andreia C. S.; VIEIRA, Gisele L. C.; TORRES, Heloisa C. A proposta da educação permanente em saúde na atualização da equipe de saúde em diabetes mellitus. Revista da Escola de Enfermagem da Universidade de São Paulo, São Paulo, v. 44, n. 2, p. 531-537, 2010.

SANTOS, Hellen G. et al. Mortalidade infantil no Brasil: uma revisão de literatura antes e após a implantação do Sistema Único de Saúde. Pediatria (São Paulo), v. 32, n. 2, p. 131-143, 2010.

SOUZA, Daniela C. et al. Disponibilidade de unidades de terapia intensiva pediátrica e neonatal no município de São Paulo. Jornal de Pediatria, Rio de Janeiro, v. 80, n. 6 , p. 453-460, 2004.

WORLD HEALTH ORGANIZATION. Patient Safety. More than Words: Conceptual Framework for the International Classification for Patient Safety: version 1.1. Final Technical Report. WHO, Jan. 2009.

Recebido em 08/02/2012

Aprovado em 15/02/2013 